Europe's Journal of Psychology 2/2009, pp. 37-40

www.ejop.org

\title{
The Efficacy of Cognitive Behavioral Couple's Therapy (CBCT) on Marital Adjustment of PTSD-diagnosed combat veterans
}

\author{
Khodabakhsh Ahmady \\ Gholamreza Karami \\ Sima Noohi \\ Arastoo Mokhtari \\ Hamide Gholampour \\ Ali-Akbar Rahimi
}

Behavioral Sciences Research Center, Baquiatallah University of Medical Sciences

Abstract

Background: Impaired marital adjustment has been introduced as one major complication of PTSD. There are controversial results about the role of Cognitive Behavioral Couple's Therapy (CBCT) on improvement of marital adjustment of PTSD veterans; the efficacy of CBCT on marital adjustment improvement is questioned in some studies and also there are no similar researches about the affect of CBCT on marital adjustment, conducted among Middle Eastern PTSD veterans. These factors motivated us to perform and evaluate our CBCT protocol among Iranian combat veterans. Methods: we enrolled 60 PTSD veterans with low degrees of marital adjustment. They were randomly distributed into intervention and control groups. Both groups have been evaluated for marital adjustment by means of ENRICH scale before and after intervention and the results were analyzed by MANOVA test. Results: The analysis was based on difference of $\mathrm{T} 2$ and $\mathrm{T} 1$ (T2-T1) where the mean of T2-T1 was -2.63 for control group and 44.9 for intervention group, sum of squares $69552.7, f=95.4$ and sig. $<000.1$. Conclusion: this shows that the prevalence of marital maladjustment is high among PTSD veterans (43\%) and the efficacy of CBCT was significant both for veterans themselves and their spouses.

Introduction

Post traumatic stress disorder is one of the major psychiatric disorders among combat veterans. Various studies have shown that $9 \%$ to $24 \%$ of war veterans suffer from PTSD. PTSD by itself has potentially symptoms that may deteriorate the quality 
of life among veterans and their family members including their partners or spouses. Apart from PTSD common symptoms, there are some complications that play a major role in their lifestyle and family life. One of the most common manifestations of PTSD is impaired marital adjustment and chronic marital distress. In studies of Solomon, the relationship between PTSD and marital intimacy among war veterans has been highlighted (Solomon et al., 2008). In a study that was performed among male American soldiers who returned from a military deployment to Iraq and Afghanistan and their female spouses or partners, the impact of individual trauma symptoms on relationship satisfaction has been studied. Their results suggest that individual trauma symptoms negatively impact relationship satisfactions in military couples in which the husbands has been exposed to war trauma (Goff et al., 2007). In a research 179 couples who were seeking relationship therapy at a veterans' affairs clinic in USA have been studied. Domestic violence rates were much higher in combat related PTSD group than the control group (Sherman et al., 2006). One of our previous studies explained that sexual dysfunction as a component of marital maladjustment is significantly associated with PTSD (Ahmadi et al., 2006). It can be realized that marital satisfaction as well as relationship intimacy and practice of violence in relationships are major co morbidities in PTSD patients. On the other hand the role of cognitive behavioral therapy (CBT) appears now to be quite important in management of marital maladjustment of PTSD patients. One of its widely used modalities is cognitive behavioral couple's therapy (CBCT).

Cognitive behavioral couple's therapy refers to cognitive therapy sessions that focus on the patient and his/her partner and their inter-personal relationship helping them to solve their problems; in the same time it supplies the couple with behavioral strategies which can help them to come up with their possible future complexes. In other words, the main goal of this method is not only problem solving but instructing the couple how they can confront new problems. Different aspects of CBCT such as education about PTSD, communication training, anger control and problem solving can improve the marital relationship of veterans and their partners (Details of our CBCT protocol is discussed in methods). In one study couples in which the husband was diagnosed with PTSD secondary to Vietnam combat experiences were subject to CBCT. After the completion of treatment, veterans reported substantial improvement in their PTSD symptoms. Although their female partners reported improved relationship satisfaction, the veterans' degree of satisfaction was unchanged across the treatment (Monson et al., 2004). In another study the role of CBCT was compared between a group of PTSD patients with co morbid substance use disorder (SUD) and isolated substance use disorder. 
The study revealed that after completion of CBCT, the PTSD group had improved relationship satisfaction and reduction in drinking and male to female violence (Rotunda et al., 2008). There are also studies which had been performed among US service members who returned from Afghanistan that illustrate the role of integrative behavioral couple's therapy (IBCD) in conflict reduction and intimacy improvement of combat veterans (Erbes et al., 2008).

As mentioned previously, cognitive behavioral couple's therapy is a modality of treatment for relationship and marital satisfaction of PTSD veterans. There is little evidence about the efficacy of this method (CBCT) in Middle Eastern combat veterans. On the other hand the efficacy of СBCT is still controversial in improvement of veterans' relationship satisfaction. As mentioned earlier in Monsen's study which was conducted among American soldiers in Vietnam, the CBCT method may improve the PTSD symptoms in veterans without any effects on veteran's marital satisfaction. Also it is not enough clear if the method can affect both the veteran and his spouse equally.

These factors besides the interest in studying our own $\mathrm{CBCT}$ protocol motivated us to study both the prevalence of marital maladjustment in PTSD veterans and the efficacy of CBCT in improving relationship satisfaction among Iranian PTSD veterans.

\section{Materials and Methods}

The study was performed as a randomized clinical Trial (RCT) and in the same time descriptive. We intended to realize the prevalence of marital dissatisfaction among PTSD veterans in the descriptive component and the efficacy of CBCT was studied in a randomized clinical trial study. For our study we enrolled all combat veterans of Iraq - Iran war who had documented history of PTSD and had been admitted to Tehran's two major veterans' affairs clinics, Baqiyatallah and Sadr hospitals, between 2007 and 2008.

The inclusion criteria were: a) being a documented veteran with post traumatic stress disorder and b) having been married at least for 3 years. We excluded veterans with spinal cord injuries or any other injuries that let them prone to sexual dysfunction. Opium addict individuals were also excluded from the study.

A number of 100 veterans with diagnosed PTSD and their spouses were subject to ENRICH marital adjustment test (Olson 1993; Fowlers 1993), where 60 couples 
gained scores below 175 which means not having optimum marital satisfaction. These 60 couples were the sample group across the clinical trial. The couples were randomly distributed in 2 groups; intervention has been performed by means of 16 to 18 sessions of $\mathrm{CBCT}$ in a 6 month period. The CBCT protocol consists of five levels and the main goals of the method is achieved at the end of $5^{\text {th }}$ level. First level (3 sessions) goes to orientation and general evaluation of the patient and his partner. On the second level (2 sessions) education of the couple is the main idea; in this phase the couple learns about PTSD and its complications, helping them to know their expectations. The third level refers to communication training and is performed in 3 sessions. The partners learn how to express their feelings and ideas.

The forth level (2 sessions) focuses on anger control and helps the couple to use anger in a positive manner. The last but not the least level is associated with problem solving abilities and needs 6 to 8 weeks in order to supply the couple with strategies and techniques of confronting new possible problems. Partners try to practice the techniques between the sessions and talk about their outcomes. The assessment of the extent of marital dissatisfaction was performed using ENRICH scale (Olson 1993; Fowlers 1993). This consists of 115 questions about different aspects of marital adjustment and includes conventionality, marital satisfaction and its subgroups. Marital satisfaction has 10 different parameters including personality issues, marital communication, conflict resolution, financial management, sexual relationship, leisure activities, parenting, friends, equalitarian role and religious orientation. We tried to work on all components of marital satisfaction but as the main idea of study implies, marital communication, conflict resolution ability, personality issues and sexual relationships were highlighted across the study. In fact we intended to evaluate these very fine inter personal issues during the study and also to assess our main goal of conflict resolution. In other words, financial management for example was not so important for us during our $\mathrm{CBCT}$ sessions as conflict resolution was because the couple's chief complaints were not the financial management but their inter-personal conflicts. The validity index for this questionnaire was $0.85-0.95$ which is an acceptable level of validity (Fowlers, 1989). In order for assessing the severity of PTSD, PTSD check list (PCL) was applied. The PCL-90-R is arranged according to DSM-SV-TR criteria for PTSD (American psychiatric association, 2005) and includes 17 sections, where the severity of symptoms can be validated in each of these 17 aspects. Kronbach's alpha is 0.87 for this questionnaire. The veterans in both groups have been assessed for marital satisfaction and PTSD before and after taking the CBCT protocol. Manova test has been performed for post-test and pre-test differences to evaluate the efficacy of intervention. All data were analyzed using SPSS. 
Results

Our results are reported in three major sections. Demographic statistics come first. Then the prevalence of marital dissatisfaction would be reported as the outcome of the descriptive component of our study and finally the results of our intervention will be discussed.

Concerning demographic data (Table 1), 60 couples were studied, and the mean age of veterans was (41.4 \pm 4.2 ) years. The minimum age was 34 and the maximum age was 54 years old. The average years of being married was $17.5 \pm 5.9$ years. The mean age of veterans' spouses was $36.5 \pm 5.4$, where the minimum and maximum were 29 and 51 respectively. The mean duration of attending the warfront was 35.7 months and the mean PTSD score of veterans according to PTSD check list was 27 with the minimum of 15 and maximum score of 46 .

Table 1. Demographic data

\begin{tabular}{|c|l|l|l|l|}
\hline & Minimum & Maximum & Mean & $\begin{array}{l}\text { Standard } \\
\text { Deviation }\end{array}$ \\
\hline $\begin{array}{c}\text { Veteran's Age } \\
\text { ( years) }\end{array}$ & 34 & 54 & 41.4 & 4.2 \\
\hline $\begin{array}{c}\text { Spouse's Age } \\
\text { ( years) }\end{array}$ & 29 & 51 & 36.5 & 5.4 \\
\hline $\begin{array}{c}\text { Marriage duration } \\
\text { (years) }\end{array}$ & 3 & 36 & 17.5 & 5.9 \\
\hline $\begin{array}{c}\text { Warfront } \\
\text { Attendance(months) }\end{array}$ & 2 & 120 & 35.7 & 25.7 \\
\hline $\begin{array}{c}\text { PTSD severity } \\
\text { (PTSD index) }\end{array}$ & 15 & 46 & 27 & 9.8 \\
\hline
\end{tabular}

About the general prevalence of marital dissatisfaction among PTSD veterans, the results indicate that of all 100 PTSD diagnosed veterans, 11 had severe marital dissatisfaction, 32 veterans had moderate marital dissatisfaction and 55 veterans reported to be relatively satisfied with their marital relationships. Only 2 veterans had optimum marital satisfaction where none of the veterans enjoyed superior marital satisfaction (Table 2). This illustrates that about $43 \%$ of PTSD veterans are absolutely dissatisfied with their marital relationship and 55\% percent have borderline marital satisfaction. 
Table 2. Marital adjustment among veterans and their spouses ${ }^{1}$

\begin{tabular}{|l|c|c|c|}
\hline Degree of Marital Satisfaction & Veterans & Spouses & Sum \\
\hline Severe Dissatisfaction & 11 & 11 & 22 \\
\hline Not Satisfied & 32 & 34 & 66 \\
\hline Borderline Satisfaction & 55 & 51 & 106 \\
\hline Optimum Satisfaction & 2 & 4 & 6 \\
\hline Superior Satisfaction & 0 & 0 & 0 \\
\hline Sum & 100 & 100 & 200 \\
\hline
\end{tabular}

Table 3. Scores of different components of Enrich marital scale among veterans ${ }^{2}$

\begin{tabular}{|c|c|c|}
\hline Maladjustment rank & Type of adjustment & Mean score \\
\hline 1 & Mutual communication & 11.62 \\
\hline 2 & Conflict resolution & 12.17 \\
\hline 3 & Financial management & 12.88 \\
\hline 4 & Parenting & 13 \\
\hline 5 & Personality issues & 13.18 \\
\hline 6 & Family and friends & 13.43 \\
\hline 7 & Sexual relationship & 13.48 \\
\hline 8 & Leisure activities & 14.02 \\
\hline 9 & Religiosity & 15.10 \\
\hline
\end{tabular}

Table 4. Showing no statistically significant difference between the two groups regarding demographic data

\begin{tabular}{|l|l|l|l|l|l|}
\hline Variable & Groups & Mean & S.D. & t-score & Sig. \\
\hline Veteran's Age (years) & Control & 43.93 & 5.6 & 1.19 & 0.24 \\
& Intervention & 42.50 & 4.2 & & \\
\hline Spouse's Age (years) & Control & 38.50 & 6.4 & 0.36 & 0.72 \\
& Intervention & 37.94 & 6.1 & & \\
\hline Marriage duration (years) & Control & 17.27 & 6.8 & 0.01 & 0.99 \\
& Intervention & 17.25 & 5.1 & & \\
\hline Warfront Attendance(months) & Control & 27.8 & 17.5 & 1.25 & 0.21 \\
& Intervention & 35.7 & 20.1 & & \\
\hline
\end{tabular}

${ }^{1}$ Of all 100 veterans and their spouses, 60 veterans and their partners were entered into the study following their low scores in ENRICH test. The table refers to the general prevalence and degree of marital dissatisfaction among war veterans.

2 The scores refer to pre-test conditions. It is shown that the mutual communication and conflict resolution are the most problematic components of marital adjustment among the veterans. 
Considering the outcome of a 6-month course of cognitive behavioral couple's therapy on 30 veterans and their spouses and comparing them to the control group shows that $\mathrm{CBCT}$ is significantly useful for the improvement of marital satisfaction among PTSD veterans. The CBCT course improved all 9 aspects of marital satisfaction with significance values of $\mathrm{P}<0.0001$ among veterans and their spouses but improvements were equal both in veterans and their partners and there were no significant difference $(P=0.23)$ The difference of Post-test and Pre-test (Post-test minus Pre-test) of mean marital satisfaction scores was the basis of our analysis. The mean difference of $\mathrm{T}_{2}-\mathrm{T}_{1}$ was $(-2.63)$ in control group where the mean difference of $T_{2}-T_{1}$ was 44.9 in intervention group. The sum of squares was 69552.7 with $d(f)=95.4$ and $P<0.0001$ (Table 5, 6). The results indicate that the efficacy of $\mathrm{CBCT}$ on marital satisfaction of PTSD patients is significant.

Considering the factor of sex on degree of marital satisfaction improvement after taking the $\mathrm{CBCT}$ course reveals that there is no significant difference between veterans and their spouses from this point of view $(P=0.23)$ (Table 7).

The study also illustrates the efficacy of $\mathrm{CBCT}$ on different components of marital satisfaction; The $\mathrm{P}$ value was $<0.0001$ for personality issues, mutual communication, conflict resolution, financial issues, leisure activities and also sexual relationship.

Table5. Mean of (T2-T1) in control and intervention groups

\begin{tabular}{|c|c|c|}
\hline GROUPS & Mean of T2-T1 & St. Dev. OF T2-T1 \\
\hline CONTROL & -2.63 & 8.4 \\
\hline INTERVENTION & 44.9 & 38.2 \\
\hline
\end{tabular}

Table 6. (MANOVA) Efficacy of CBCT on marital satisfaction (total score)

\begin{tabular}{|l|l|l|c|c|c|}
\hline Variable & Sum of squares & Mean of squares & $d f$ & $f$ & Sig. \\
\hline $\begin{array}{l}\text { CBCT efficacy on marital } \\
\text { satisfaction scores }\end{array}$ & 69552.7 & 69552.7 & 1 & 95.4 & $<000.1$ \\
\hline
\end{tabular}

Table 7. Considering the factor of sex (veterans vs. spouses) the results are not significant

\begin{tabular}{|l|l|l|r|r|r|}
\hline Variable & Sum of squares & Mean of squares & \multicolumn{1}{|c|}{ df } & \multicolumn{1}{c|}{$f$} & \multicolumn{1}{c|}{ Sig. } \\
\hline Group(intervention vs. control) & 70228.4 & 70228.4 & 1 & 96.3 & $<000.1$ \\
\hline Sex (veterans vs. their partners) & 1038.4 & 1038.4 & 1 & 1.42 & 0.23 \\
\hline
\end{tabular}




\section{Discussion}

Living with a patient who is suffering from PTSD could be challenging for each of his family members especially his spouse. Re-experiencing the trauma, emotional numbing, impaired interpersonal, familial and social performance are some of their common symptoms (American psychiatric association, 2005).

Some studies even emphasize the severity of PTSD symptoms in military-associated PTSD compared with PTSD due to other etiologies (Naifeh et al., 2008). Veteran's spouse is the first individual who confronts these debilitating symptoms of PTSD. On the other hand the practice of interpersonal violence which is common among PTSD veterans can lead to PTSD and physical stress in spouses as some studies imply (Woods et al. , 2008;Dejonghe et al. , 2008).

The rate of marital maladjustment is high among PTSD veterans according to our results which are similar to those of previous studies: $43 \%$ of veterans with PTSD are absolutely dissatisfied with their marital life and 55\% have borderline satisfaction. Marital maladjustment is so serious that according to some studies marriage breakdown is inevitable among PTSD patients (Gruden, 2000).

Among nine aspects of marital adjustment including personality issues, mutual communication, conflict resolution, parenting, sexual relationship, leisure activities, family/ friends and religiosity, mutual communication were the most problematic issues where veterans gained the lowest scores. (Table 3)

This aspect of marital adjustment is related to mutual understanding, verbal and non verbal communication and generally evaluates the individual's perception of his marital life. Our previous studies among general population, also indicates that mutual communication is the most problematic axis of marital maladjustment; this reveals the fact that there is no significant difference between veterans and general population from this point of view.

The present study also pointed out that there is a relationship between PTSD severity and the degree of marital maladjustment. The more severe the PTSD is the greater dissatisfaction could be generated consequently. It can be assumed that, a vicious cycle would appear in this regard.

Improving marital satisfaction may result in discontinuation of the vicious cycle and prevent the deterioration of PTSD. Therefore studies recommend couple therapy as 
one of the most efficient modalities of treatment for PTSD patients (Monson, 2004). The cognitive behavioral couple's therapy is a simple and relatively inexpensive method and can be performed in all age groups, cultural and educational levels. The goal of CBCT is to instruct the couple how to manage and handle their present and future problems. This method can affect all components of marital adjustment positively as mentioned previously in our results.

On the other hand, in contrast to our presumptions, the CBCT was not significantly effective on improving PTSD symptoms. Maybe we need to consider a long term follow up in order to judge about improvement of PTSD symptoms. Nevertheless, it seems that we need more studies in the future to expertise about the association of CBCT and PTSD symptoms.

In conclusion, we recommend a routine evaluation of marital adjustment status in all PTSD diagnosed veterans and their spouses or partners. Finally, cognitive behavioral couple's therapy should be considered as a component of PTSD management in all patients.

\section{References}

Ahmadi k, Fathi Ashtiani a, Zareir a, Arabnia a, Amiri M (2006). Sexual dysfunction and marital adjustment in veterans with PTSD .Arch Med Sci 2006;2, 4:280-285.

American psychiatric Association, DSM IV, DRAFT CRITERIA, copy right 1993 .by the American Psychiatric Association (2005); Washington DC P. K. 8-9.

Dejonghe ES, Bogat GA, Levendosky AA, Eye A (2008).PTSD symptoms among men and women survivors of intimate partner violence: the role of risk and protective factors. J Postgrad Med. 2008 Oct-Dec;54(4):294-300.

Erbes CR, polusny MA, Macdermid S, Compton JS $(2008)$.Couple therapy with combat veterans and their partners. J Clin psychol. 2008 Aug; 64(8):972-83

Fowlers Bj, Olson DH, Enrich marital inventory (1989). A discriminate validity and cross validation assessment. J Marital fam ther 1989; 15:65-79.

Fowlers Bj, Olson DH (1993). Enrich marital satisfaction scale: A Brief research hand clinical tools. J fam Psycho 1993; 7:176-85.

Goff BS, Crow JR, Reisbig AM, Hamilton S (2007). The impact of individual trauma symptoms of deployed soldiers on relationship satisfaction J fam Psychol, 2007 Sep, 21(3):344-53. 
Gruden V, Gruden V (2000). J Libido and PTSD. Coll .Antropol 2000; 24:253-6.

Monson CM, Schnurr PP, Stevens SP, Gutgrie KA (2004). Cognitive- Behavioral Couples Treatment for posttraumatic stress disorder: initial findings. J Trauma Stress, 2004 Aug; 17(4):341-4.

Naifeh JA, North TC, Davis JL, Reyes G, Logan CA, Elhai JD(2008).Clinical profile differences between PTSD-Diagnosed military veterans and crime victims. Journal of trauma dissociation 2008; 9(3):321-34.

Olson DH, Olson A (1993). Enrich Canada Inc. J fam ministry 1993; 11:28-53.

Rotunda RJ, Ofarrell TJ, Murphy M, Babey SH (2008). Behavioral couples therapy for comorbid substance use disorders and combat-related posttraumatic stress disorder among male veterans: an initial evaluation. .Addict Behav, 2008 Jan; 33(1): 180-7. Epub 2007 Jun 9.

Sherman MD, Sautter F, Jackson MH, Lyons JA, Han X (2006). Domestic violence in veterans with posttraumatic stress disorder who seek couples therapy. J Marital Fam ther. , 2006 Oct, 32(4):479-90.

Solomon Z, Dekel R, Zerach $G$ (2008). The relationship between posttraumatic stress symptoms clusters and marital intimacy among war veterans. J fam Psychol, 2008 Oct; 22(5):659-66.

Woods SJ, Hall RJ, Campbell JC, Angott DM (2008). Physical health and post traumatic stress symptoms in women experiencing intimate partner violence. J Midwifery Women's Health. 2008 Nov-Dec; 53(6):538-46.

About the authors:

Dr. Khodabakhsh Ahmadi is associate professor at the Baquiatallah University of Medical Sciences in Tehran and he has been working on PTSD, sexual dysfunction and marital satisfaction since eleven years ago having plenty of international publications in his field.

E-mail: brc_baquiatallah@yahoo.com

Gholamreza Karami (MD), Sima Noohi (MD), Arastoo Mokhtari (MD), Hamide Gholampour (BS), and Ali-Akbar Rahimi (MS) are the academic staff of Behavioral Research Center of Baquiatallah University. 